\title{
Anabases
}

ANABASES Traditions et réceptions de l'Antiquité

$8 \mid 2008$

Varia

\section{Entretien inédit (II) : «Ce que je pensais, ce que je faisais »}

Jean-Pierre Vernant

\section{OpenEdition}

1 Journals

Édition électronique

URL : http://journals.openedition.org/anabases/93

DOI : 10.4000/anabases.93

ISSN : 2256-9421

Éditeur

E.R.A.S.M.E.

Édition imprimée

Date de publication : 1 octobre 2008

Pagination : 13-24

ISSN : 1774-4296

\section{Référence électronique}

Jean-Pierre Vernant, «Entretien inédit (II) : « Ce que je pensais, ce que je faisais » », Anabases [En ligne], 8 | 2008, mis en ligne le 01 juillet 2011, consulté le 21 octobre 2019. URL : http:// journals.openedition.org/anabases/93 ; DOI : 10.4000/anabases.93

(c) Anabases 
Anabases 8 (2008), p. 13-24.

\section{Entretien inédit (II) : \\ "Ce que je pensais, ce que je faisais"}

JEAN-PIERRE VERNANT ${ }^{1}$

\section{Le Centre Louis Gernet, le marxisme et le microcomparatisme}

José Otávio Guimarães

Est-il vrai qu'au départ le Centre Gernet ne comportait pas d'helléniste, que c'était vous seulement qui occupiez cette fonction?

\section{Jean-Pierre Vernant}

Le Centre Louis Gernet a son origine dans le comparatisme, puisque, avant qu'il ne soit le Centre de recherches comparées sur les sociétés anciennes, l'origine de ce Centre, ça a été le fait qu'au début des années soixante, un certain nombre d'historiens, d'antiquisants, d'anthropologues, marxistes en général, se sont réunis pour travailler dans une perspective comparatiste.

\section{José Otávio Guimarães}

Juste un éclaircissement : vous dites « marxistes en général »... Le Centre Gernet est-il issu du Centre d'études et de recherches marxistes ${ }^{2}$ ?

1 Entretien avec José Otávio Guimarães. Sèvres, octobre 2000. Toutes les notes sont de l'interviewer.

2 Je me suis appuyé pour poser cette question sur les mots suivants de Giulia SISSA, "Philology, anthropology, comparison : the French experience", Classical Philology 92 (1997), p. 169 : "Before its present name, Centre Louis Gernet, the group of scholars around Jean-Pierre Vernant was called Centre de Recherches Comparées sur les Sociétés Anciennes. Even earlier, from 1962 to 1965, the very first core of what would become the "équipe" was constituted of a small group or cell of researchers - namely, the Indianist Louis Dumont, the Sinologist Jacques Gernet, the Sumerologist René Labat, the ethno- 


\section{Jean-Pierre Vernant}

Non. Celui-ci n'a rien à voir avec le Centre des recherches comparées sur les sociétés anciennes; ça, c'était le centre marxiste officiel contrôlé par le PC, avec lequel nous avions des relations plutôt difficiles.

\section{José Otávio Guimarães}

Quand vous dites «nous », vous parlez de quelles personnes exactement?

\section{Jean-Pierre Vernant}

Les gens avec lesquels j'ai pris langue pour constituer un groupe informel, qui comprenait : un helléniste, moi ; un spécialiste de Rome, Jean-Paul Brisson ; des spécialistes du monde babylonien, Paul Garelli, Elena Cassin, Jean Bottéro ; un spécialiste de l'Égypte, Jean Yoyotte; un spécialiste de l'Inde, Paul Lévy et ensuite Charles Malamoud; des africanistes, très vite Maurice Godelier ; des spécialistes du monde éthiopien, les Tubiana, lui et sa femme ; un sémitisant.

\section{José Otávio Guimarães}

Peut-on dire tout de même qu'il y avait dans ce groupe une orientation marxisante?

\section{Jean-Pierre Vernant}

Tous ces gens s'étaient imprégnés de marxisme, mais ils trouvaient que le marxisme, tel qu'il était officiellement proposé, c'était une caricature ${ }^{3}$. D'autre part, on

logist Jean Guiart, in addition to a few classicists - meeting regularly at the Centre d'Études et de Recherches Marxistes (CERM). This political framework is of course a relevant aspect of the entire enterprise, as we shall see. In 1965, Fernand Braudel, the president of the École Pratique des Hautes Études en Sciences Sociales et Économiques, made possible the foundation of the Centre the Recherches Comparées sur les Sociétes Anciennes. Marcel Detienne, recently named Chef de Travaux at the École, was in charge of its direction, with Jean-Pierre Vernant. Classicists like Pierre Vidal-Naquet, Jean-Paul Brisson, Claude Nicolet, Moses I. Finley, and the original group of CERM joined Detienne and Vernant". Pour les rapports de J.-P. Vernant à ces caricatures, voir, par exemple, "Lantiquité esclavagiste", La Pensée 77 (1958), p. 80-89 ; "La lutte des classes", Eirene - Studia Graeca et Latina IV (1965), p. 5-19, où il écrit : "Pour les marxistes, en effet, le monde ancien constitue une société de classes qui peut être définie dans sa forme typique comme le mode de production esclavagiste. Mais peut-on en conclure sans autre précaution que l'histoire de l'Antiquité classique tout entière doit être considérée comme le champ où se seraient affrontées les deux classes antagonistes des esclaves et des propriétaires d'esclaves? Si la théorie marxiste devait se réduire à une formule aussi sommaire, aussi figée, aussi antidialectique, elle ne serait guère susceptible d'éclairer le travail des historiens ". 
avait affaire à toute une série de civilisations et d'époques auxquelles Marx ne s'était pas intéressé : archaïques, orientales, etc. Il est vrai qu'il y a eu des réunions où on avait remis en avant les textes de Marx sur le despotisme oriental ; mais Marx connaissait ça de seconde main, par la lecture de quelques indianistes. Ce n'était pas son affaire. La Grèce et Rome, il connaissait, mais il n'a jamais pris cela comme thème de recherche. D'une certaine façon, ni l'Égypte ancienne ni le monde babylonien n'existaient encore à son époque ; le suméro-acadien n'était pas encore sorti. Nous étions donc des marxisants mais qui travaillaient avec des documents que les marxistes n'avaient pas regardés.

\section{José Otávio Guimarães}

Pouvez-vous nous raconter plus ou moins comment se déroulait ce travail ?

\section{Jean-Pierre Vernant}

Ce n'était pas facile. Nous prenions les grands faits humains qu'au fond on retrouve dans toute société. Par exemple, le rapport à la terre : sédentaire, agriculteur, pasteur, nomade; le problème de la ville, des villes, des grandes agglomérations urbaines; le problème du pouvoir : royauté, hiérarchie ; le problème de la guerre: conflit, à l'intérieur et à l'extérieur ; des problèmes de religions : le sacré, le culte, les mythes ; l'économie : la production de richesses, la circulation, les formes de l'échange. Tous ces problèmes-là, ils se posent pour toute collectivité humaine, mais ils revêtent des faciès très différents dans les diverses sociétés. Alors, on disait bon, on va mettre le pouvoir, la royauté et alors l'Égyptien, le Sumérien, le Grec, le Romain, l'Indien, l'Africain faisait un topo où il expliquait comment le problème se posait pour lui. Et on discutait. Comme je l'ai dit trente-six fois, le résultat de cela n'était pas simplement de nous apprendre des choses sur d'autres cultures que celle sur laquelle nous travaillions, mais surtout on ne pouvait plus regarder la culture sur laquelle on travaillait de la même façon, après avoir vu comment c'était ailleurs.

\section{José Otávio Guimarães}

On part ailleurs et puis on rentre chez soi transformé pour se regarder d'une autre manière?

\section{Jean-Pierre Vernant}

Bien entendu. On a travaillé comme ça pendant quatre ou cinq ans. On publiait à la fin de l'année des résumés. Qu'est-ce qu'ils sont devenus ? Je n'en sais rien. Je les ai eus à un moment donné. Dans le bordel, ça a disparu.

\section{José Otávio Guimarães}

Mais quelques-unes de ces recherches ont été publiées? 


\section{Jean-Pierre Vernant}

Oui, celles sur la terre, la guerre, la divination, la religion, la mort et le corps humain ${ }^{4}$. On arrive à six volumes comparatistes que nous avons faits.

\section{José Otávio Guimarães}

Aujourd'hui, pourtant, le Centre est particulièrement connu pour les recherches menées par ses hellénistes...

\section{Jean-Pierre Vernant}

Il y a eu des changements dans le Centre. En effet, des choses se sont passées. En 1964, on dépensait de l'argent, on cotisait, on n'avait pas d'argent, on n'avait pas de local. Il y avait beaucoup de difficultés. Il y avait l'École pratique des hautes études où j'étais, qui créait des Centres. On m’a dit: "Tu devrais créer un centre d'études de recherches comparées. " J'ai donc créé ce Centre. Et au départ, dans ce Centre, il y avait tous ces gens que je viens de citer. Mais il y a eu un afflux d'hellénistes: Marcel Detienne, Pierre Vidal-Naquet et tous les autres qui sont arrivés. Et alors l'équilibre a basculé du côté de l'hellénisme. Quand, en 1984, j’ai eu la médaille d'or du CNRS et que j'ai fait un topo sur le Centre, j'ai dit que je souhaitais que le centre continue d'être comparatiste ${ }^{5}$. Et trente-six fois au cours des assemblées $j$ 'ai dit qu'il fallait reprendre la perspective comparatiste. Mais moi, j’ai 87 ans, ce n'est pas à moi de le faire.

\section{José Otávio Guimarães}

On parle, à présent, non seulement de comparatisme, mais, dans le domaine des études de la religion grecque, de microcomparatisme. En ce qui concerne vos travaux, que pensez-vous de ce changement d'échelle?

\section{Jean-Pierre Vernant}

Si l'on prend ce livre que j'appelle La mort et les morts dans les sociétés anciennes, et si l'on regarde cette espèce d'introduction comparative que j'ai faite ${ }^{6}$, c'est vraiment du

4 M. FinLEY (sous la direction de), Problèmes de la terre en Grèce ancienne, Paris-La Haye, Mouton, 1973 ; J.-P. VERNANT (sous la direction de), Problèmes de la guerre en Grèce ancienne, Paris, Mouton-EPHE, 1968 ; Divination et rationalité, Paris, Le Seuil, 1974 ; La cuisine du sacrifice en pays grec (avec M. DETIENNE), Paris, Gallimard, 1979; La mort, les morts dans les sociétés anciennes (avec G. GNOLI), Cambridge-Paris, Cambridge University Press-Éditions de la Maison des Sciences de l'Homme, 1982 ; Corps des Dieux (avec Ch. MALAMOUD), Le temps de la réflexion 7 (1986).

5 J.-P. VERNANT, "Chercheur au CNRS" (Discours prononcé le 18 décembre 1984, à l'occasion de la remise de la médaille d'or du CNRS), in Entre mythe et politique, Paris, Le Seuil, 1996, p. 43-49.

6 Cette introduction a été reprise par J.-P. VERNANT sous le titre "Inde, Mésopotamie, Grèce : trois idéologies de la mort", in L'individu, la mort, l'amour (1989), Paris, Gallimard (Folio/Histoire), 1996, p. 103-115. 
microcomparatisme. Pourquoi ? Parce que je prends la mort babylonienne, la mort indienne - de l'Inde ancienne - et la mort grecque, et je les prends sous la forme des funérailles. Je prends un petit détail, un tout petit détail : à savoir que les Grecs et les Indiens brûlent les cadavres. Mon texte, c'est sur le monde homérique, sur l'image qu'il s'est fait de la mort. Dans les deux cas, c'est pareil, on les brûle, contrairement aux Assyro-Babyloniens qui ne les brûlent pas du tout, qui les mettent en terre, souterrainement, étendus, avec de quoi bouffer, comme si la mort c'était une vie dans le fond de la terre. Et que les morts étaient les racines des vivants. Donc, c'est pareil, sauf un détail. Quand les Grecs brûlent le cadavre, comme il est dit de la façon la plus explicite, ils éteignent l'ardeur du feu avec du vin et de l'eau et ils repèrent dans les cendres les os blancs calcinés, très visibles, dit le texte, dans les cendres. Et ils les repèrent pour les prendre, pour les mettre dans un réceptacle, peut-être en y mettant de l'huile; ils recueillent les ossements et quand ils ont recueilli les ossements brûlés, ils les mettent en terre et plantent un sema, un signe. Petit détail : les Indiens brûlent le cadavre de la même façon, quand le feu est éteint, ils recueillent les ossements et ils les brûlent une seconde fois. C'est ce qu'on appelle le système de seconde crémation. Et quand tu tires sur ce petit détail, tu amènes tout. C'est-à-dire tu vois que n'est pas seulement la mort qui est vue autrement, c'est toute la civilisation qui est orientée dans un autre sens.

\section{José Otávio Guimarães}

Ne croyez-vous pas qu'il y a des limites « objectives » à l'application d'un comparatisme généralisé ? Lorsqu'on étudie la Grèce ancienne, cela impose certaines contraintes épistémologiques spécifiques, différentes, par exemple, de celles imposées par l'étude des Bororos.

\section{Jean-Pierre Vernant}

Oui, tu as raison.

\section{José Otávio Guimarães}

Pour en revenir au Centre Louis Gernet, il n’a jamais existé, à mon avis, d' «École de Paris ", comme le disent les hellénistes anglo-saxons ${ }^{7}$, mais il y a eu quand même un mouvement, une manière française de faire de l'anthropologie de la Grèce antique,

7 Sur l'idée anglo-saxone d'une "École de Paris", voir, par exemple, R. BUXTON, "Introduction", in R. L. GORDON, Myth, Religion and Society - Structuralist Essays, Cambridge, Cambridge University Press, 1981, p. ix-xvii ; Ch. SEGAL, "J.-P. Vernant and the study of ancient Greece", Arethusa 15 (1982), p. 221-234; J. RedFIELD, "J.-P. Vernant: Structure and History", History of Religions, XXXI, 1 (1991), p. 69-74; O. Murray, "Pierre Vidal-Naquet et le métier d'historien de la Grèce : l'École de Paris", in F. Hartog, P. SChMiTT et A. SCHNAPP, Pierre Vidal-Naquet, un historien dans la cité, Paris, La Découverte, 1998, p. 154-166. 
un travail collectif qui a produit de très beaux fruits. Et le Centre Gernet en a été le locus institutionnel.

\section{Jean-Pierre Vernant}

Tout à fait, chacun mène sa barque ; mais tout de même, chacun tient compte aussi de ce que fait l'autre et parle avec lui, évidemment.

\section{José Otávio Guimarães}

Mais il y a quand même un débat de fond qui est commun. Que faire de l'anthropologie historique au sein même de ce mouvement ? Je crois qu'il y a des propositions différentes. Ce que fait Vidal-Naquet, par exemple, avec une approche plus historique, est différent de ce que vous faites vous, dans une perspective plus philosophique ${ }^{8}$, ou encore de ce que fait Detienne dans une perspective plus "philologique ".

\section{Jean-Pierre Vernant}

Moi, si tu veux, j'étais beaucoup plus proche de Marcel Detienne dans mes procédures intellectuelles, dans la période où lui et moi on s'occupait des mythes. Il était très proche de moi ; et moi très proche de lui. Parce qu'il avait des qualités, des connaissances que je n'avais pas, et moi j’avais aussi des qualités, je ne dirais pas des connaissances, mais des qualités que lui n'avait pas ; des qualités peut-être d'invention.

\section{Chez les historiens}

[Alors que nous sortions de la salle à manger pour nous rendre dans le salon, le magnétophone sest arrêté. Je venais de lui poser une question sur ses rapports institutionnels avec les historiens de la mouvance des Annales, et plus particulièrement sur le processus qui l'avait

P. VIDAL-NAQUET a lui-même remarqué les différences de son travail par rapport à la perspective de la psychologie historique vernantienne : «mon travail d'historien s'apparente bien à ce qu'Ignace Meyerson et Jean-Pierre Vernant ont appelé la "psychologie historique", mais nos voies ont été différentes. Meyerson et Vernant sont partis des catégories psychologiques, dont ils ont montré qu'elles n'étaient pas éternelles, et ont rencontré - parce qu'ils les cherchaient - les textes et les institutions politiques et sociales. J'ai accompli le cheminement inverse ", Le chasseur noir. Formes de pensées et formes de société dans le monde grec (1981), Paris, La Découverte, 1991, p. 15. Différences que J.-P. Vernant aussi ne cessait de souligner : "Philosophe, j'ai tendance à chercher les grandes lignes, à m’interroger sur le sens : qu'est-ce que cela veut dire plutôt que comment cela est-il advenu, lecteur et interprète de textes plutôt que découvreur et déchiffreur de documents. [...] Il est vrai que j'essaie de comprendre sans être habité de cette "rage historienne" qui possède Vidal et qu'il m’est arrivé de lui envier ", "Postface", in F. HarTOG, P. SCHMiTT et A. SCHNAPP, op. cit., p. 220-221. Sur la démarche historienne de Vidal-Naquet, voir le très beau livre de F. HARTOG, Vidal-Naquet, historien en personne - L'homme-mémoire et le moment-mémoire, Paris, La Découverte, 2007. 
conduit à obtenir, en 1958, le poste de directeur d'études à la VIe Section de l'École Pratique des Hautes Études qui venait juste d'être créée.]

\section{Jean-Pierre Vernant}

[...] À ce moment-là, il fallait avoir l'approbation de Braudel. Il était le monarque qui décidait de tout. Et puis, il y avait tous les premiers membres de l'École qui ne vous connaissaient pas, ou qui vous connaissaient mal sur le plan scientifique. Gernet me dit : « Pour être candidat, tu dois avoir l'accord de Braudel. Il a un séminaire, où il fait parler d'autres gens que lui. Tu vas lui proposer d'occuper son séminaire pendant une heure ». Je fais donc, chez Braudel, un exposé sur le travail et la nature en Grèce ancienne, qui est paru après sous la forme d'un article, et qui portait sur l'absence de la catégorie économique et psychologique du travail dans le monde hellénique ${ }^{9}$.

\section{José Otávio Guimarães}

Juste une curiosité : André Aymard, qui, parmi les historiens de l'Antiquité, écrivait sur le thème du travail en Grèce ${ }^{10}$, était-il présent ?

\section{Jean-Pierre Vernant}

Non. Aymard à ce moment-là était doyen à la Sorbonne, professeur d'histoire ancienne. C'est vrai qu'il avait participé au colloque organisé par Meyerson en 1941, à Toulouse, sur le travail et les techniques. Aymard était intervenu sur le travail, mais pas dans une perspective comme celle de la psychologie historique. Le Journal de psychologie a publié en 1948, après la fin de la guerre, l'article d'Aymard et ceux de tous les autres intervenants ${ }^{11}$.

\section{José Otávio Guimarães}

Oui, Lucien Febvre a envoyé un texte, Marcel Mauss aussi, et Marc Bloch, après un voyage difficile de Clermont-Ferrand à Toulouse, a pu participer personnellement à ce colloque ${ }^{12}$.

$9 \quad$ Voir la note 14.

10 A. Aymard, "Hiérarchie du travail et autarcie individuelle dans la Grèce archaïque", Revue d'histoire de la philosophie et d'histoire générale de la civilisation 11 (1943), p. 124-146.

11 A. Aymard, "L'idée de travail dans la Grèce archaïque", Journal de psychologie normale et pathologique (JDPs) 41 (1948), p. 29-45.

12 Sur ce colloque, voir R. Di DONATO, Per una antropologia del mondo antico, Firenze, La Nuova Italia, 1990, p. 153-154, où l'on peut lire le commentaire suivant : "Al di là dell'interesse che acquista nella nostra ricostruzione, l'insieme dei testi che compongono il colloquio costituisce a parer mio un importante documento di un momento della storia culturale francese. Esso sanziona continuità e fratture, arricchimenti e perdite senza riparo. L'incontro pieno tra il metodo comparativo della psicologia storica e la prima generazione degli storici delle Annales avviene poco prima del termine dell'esperienza comun tra i due fondatori. " 


\section{Jean-Pierre Vernant}

Tout à fait. J'ai fait donc mon topo chez Braudel. Beaucoup de monde. Tous les directeurs d'études. Il y avait des étudiants, mais essentiellement tous les directeurs d'études venant voir qui était ce type-là. Quand j'ai fini, Braudel était très réticent. Et alors Gernet prend la parole et fait un éloge. Quelques directeurs d'études prennent ensuite la parole, en particulier Louis Dumont, pour dire : c'est épatant, c'est remarquable, exactement ce qu'il faut faire. Et puis, effectivement, Braudel présente ma candidature ${ }^{13}$.

\section{José Otávio Guimarães}

Les gens ne connaissaient pas ce que vous aviez écrit sur la Grèce ?

Jean-Pierre Vernant

J'avais très peu publié.

\section{José Otávio Guimarães}

Il y avait, par exemple, l'article de 1952 sur "Prométhée et la fonction technique $^{14 "}$ ?

13 F. Braudel jouera également un rôle important pour qu'Ignace Meyerson, lui aussi, puisse être nommé directeur d'études à la $\mathrm{VI}^{\mathrm{e}}$ Section de l'EPHE. Rappelons, ici, l'anecdote racontée, ailleurs, à ce sujet par J.-P. VERNANT : "Les historiens l’ont pour la plupart ignoré. [...] Les historiens de l'école des Annales étaient des anti-Seignobos. Or Meyerson avait dédié sa thèse à Seignobos, avec qui il était très lié. Braudel ne le lui a jamais tout à fait pardonné. À l'époque où Meyerson était à Toulouse, celui-ci me demanda de rendre visite à Braudel. C'est ce que je fis, en 1948, afin de plaider la cause de Meyerson, d'entretenir l'historien de travaux en cours dans le domaine de la psychologie. Cela ne l'intéressait pas beaucoup ; c'est d'ailleurs évident quand on lit ses premiers ouvrages. Gernet était intervenu pour que Braudel prenne Meyerson à l'École. La grande "affaire", c'était ça. Finalement, Meyerson a été nommé en 1951 ", "Lire Meyerson", in Entre mythe et politique, p. 158-159.

14 En fait, jusqu'à la fin de 1957, J.-P. VERNANT n'avait publié, dans le domaine des études sur la Grèce antique, que cinq articles: "Prométhée et la fonction technique", JdPs 45 (1952), p. 419-429; "Travail et nature en Grèce ancienne", JdPs 52 (1955), p. 1-29; "Aspects psychologiques du travail dans la Grèce ancienne", La Pensée, 66 (1956), p. 8084 ; "Remarques sur les formes et les limites de la pensée technique chez les Grecs", Revue d'Histoire des Sciences 10 (1957), p. 205-255 ; "Du mythe à la raison. Recherches sur la formation de la pensée positive en Grèce archaïque", Annales E.S.C., XII, 2 (1957), p. 183-203. Les quatre premiers articles iront constituer, quelques années plus tard, le dossier "Le travail et la pensée technique" de Mythe et pensée chez les Grecs. Études de psychologie historique (1965), Paris, La Découverte, 1996, p. 261-322, le dernier, paru d'ailleurs dans la revue des nouveaux historiens, clôturera ce même livre. 


\section{Jean-Pierre Vernant}

Écoute, la plupart ne me connaissaient pas. Ils se demandaient qui était ce typelà. J'avais publié, il est vrai, beaucoup de comptes rendus dans le Journal de psycholo$g^{i e}{ }^{15}$. Les gens le lisaient dans ce milieu. Mais je ne suis plus un jeunot, je suis un revenant, à cause de la guerre et de la résistance. En 1948, j’ai 34 ans ${ }^{16}$.

\section{José Otávio Guimarães}

Mais vous aviez déjà publié des articles sur la politique internationale, dans Action?

\section{Jean-Pierre Vernant}

Pendant deux ans, il est vrai, entre 1946 et 1948. Peut-être que certains le savent, mais ça n'a pas dû compter. Finalement Braudel me présente à l'assemblée. Voilà les candidats que nous avons, et puis les gens votaient. Et entre-temps, j'avais été chez Meyerson qui m’avait dit d'aller voir Lucien Febvre, qui était vieux, malade, qui ne venait plus aux réunions, mais qui, enfin, était quand même le patron de Braudel. Et c'était à l'hôpital, dans le cinquième, je ne me rappelle plus, peu importe. Il était très vieux, me semblait-il. Et puis alors, il ne me connaissait pas. Je lui avais auparavant téléphoné en lui demandant s'il pouvait me recevoir, sans trop le déranger. Il m’a peut-être donné un coup de main en intercédant auprès de Braudel et d'autres directeurs d'études qui votaient à l'assemblée ${ }^{17}$.

\section{Diderot et la philosophie durant la Résistance}

\section{José Otávio Guimarães}

Pourriez-vous me dire quelque chose sur votre mémoire de Licence consacré à la notion de vie chez Diderot ? Pourquoi, à ce moment-là, au milieu des années 1930, cet intérêt pour Diderot ${ }^{18}$ ?

15 Entre 1950 et 1957 , Vernant avait déjà publié plus de 55 comptes rendus. Ils ont été réunis par R. Di Donato dans le deuxième volume de J.-P. VERNANT, Passé et présent. Contributions à une psychologie historique, Roma, Edizioni di Storia e Letteratura, 1995. 1948 est une date important dans la vie de l'helléniste Vernant puisqu'il devient chercheur au CNRS.

17 La direction d'études de J.-P. Vernant s'intitulera Pensée sociale et religieuse de la Grèce ancienne.

18 Sans avoir pu consulter ce mémoire, je ne connaissais le Diderot de J.-P. Vernant que par l'intermédiaire du long compte rendu qu'il avait écrit sur "Le Diderot d'Henri Lefebvre", La Pensée 29 (1950), p. 89-94. 


\section{Jean-Pierre Vernant}

Je lis Diderot avec ravissement. Non seulement sa prose, qui était magnifique, mais la correspondance et les petits écrits. J'ai envie donc de faire quelque chose làdessus. La notion de vie parce qu'il est matérialiste. C'est un matérialiste mais pas mécaniste comme Descartes. Les choses ont changé depuis l'époque cartésienne, où c'est l'espace et le mouvement qui expliquent tout. Chez Diderot c'est la vie, c'est les cellules, c'est la biologie qui est bien ; en même temps c'est la notion vie en art, en politique. C'est son matérialisme vitaliste et c'est aussi la notion de vie en morale, en esthétique, et tout ça.

\section{José Otávio Guimarães}

Et n’avez-vous pas été marqué par Bergson, un maitre-penseur à ce moment-là et très attiré justement par la notion de vie?

\section{Jean-Pierre Vernant}

J'ai lu Bergson, mais le mémoire n'est pas du tout bergsonien. C'est très anti-bergsonien. Diderot ne s'occupe pas de Bergson, bien entendu; ni Bergson ne s'occupe de Diderot. C'est complètement à côté. Pour Bergson, c'est l'esprit qui d'une certaine façon fonde la matière. Pour Diderot, je dirais volontiers que c'est la matière et une certaine énergie vitale qui fondent l'esprit. Les positions sont inversées. Mais je dois l'avoir écrit/lu ? quelque part. J'ai retrouvé aussi un texte, quand j'étais candidat à l'agrégation, une dissertation sur la mort chez Spinoza; quelques mots, ce n'est pas énorme.

\section{José Otávio Guimarães}

Quand vous donniez des cours de philosophie, au Lycée de Toulouse et après au Lycée Jacques Decour, à Paris, est-ce que vous aviez un programme ? Vous vous souvenez de votre enseignement ? Vous parliez essentiellement de quoi à vos étudiants ?

\section{Jean-Pierre Vernant}

Quand je suis arrivé au Lycée de Toulouse, fin novembre 1940, ça faisait trois ans que j'étais à l'armée, à la frontière italienne, et puis, ensuite, avec l'avancée allemande, tout à fait en première ligne, ensuite ramené à Narbonne et démobilisé à Narbonne. Je n'avais pas fait de philosophie pendant trois ans. Je n'avais pas un volume. Je n'avais pas un livre. Je n'avais pas une note. Je n'avais rien. Tout était resté à Paris, où j'étais étudiant. Je n'avais rien. Et j'avais des classes non seulement de philosophie, mais aussi d'hypokhâgne et de khâgne. Très lourd. Comment je me démerdais ? Je n'en sais rien. J'improvisais tous mes cours, comme je pouvais. Meyerson m'a donné un coup de main. Comme à ce moment-là dans les classes de philosophie, il y avait une grande partie qui était la psychologie, alors je lui disais : "Voilà, j’ai fait ça ». Il me répondait : "Bon, maintenant, il faut faire ça. On a fait l'habitude, maintenant on doit faire la mémoire». Il me faisait une espèce de topo, où il m'expliquait ce qu'il y avait à dire, 
comment se présentait pour le psychologue, aujourd'hui, tel ou tel problème. Et moi je reprenais ça, à ma façon, et j'expliquais ça aux élèves. Et pour les hypokhâgneux et khâgneux, je me demande comment je faisais. J'improvisais. Je me rappelle que je faisais d'une certaine façon à tire-larigot des espèces de traversées de psychologie historique, sur la personne, sur le temps, etc.

\section{José Otávio Guimarães}

Vous ne faisiez donc pas des cours de philosophie traditionnels?

\section{Jean-Pierre Vernant}

Il y en avait un peu. J'étais obligé de faire des cours sur Descartes ou sur Kant. Je faisais aussi de la philosophie générale : l'être, le devenir, le réel. J'en faisais, mais j'improvisais tout.

\section{José Otávio Guimarães}

Et Marx ? Il ne faisait pas partie, à cette époque, des auteurs les plus considérés du point de vue philosophique. Avait-il, Marx, une place dans les cours de philosophie d'un lycée à cette époque-là ?

\section{Jean-Pierre Vernant} Vichy.

Certainement pas. Tu plaisantes! On ne pouvait pas. C'était sous le régime de

\section{José Otávio Guimarães}

Et vous faisiez en même temps de la résistance et les cours?

\section{Jean-Pierre Vernant}

Oui, pendant une période j'ai fait les deux, jusqu'au moment où j'ai appris, grâce à des lettres que j'ai reçues de Vichy, des lettres que j'ai reçues du concierge et d'un garçon qui travaillait avec le proviseur, que j'allais être arrêté. Donc là, j'ai laissé tomber le lycée. J'ai fait ce qu'on appelle... " plonger », c'est-à-dire que je suis devenu clandestin. À côté, je m'occupais d'organiser, de participer et d'emmener des groupes pour saboter ceci, faire sauter cela.

\section{José Otávio Guimarães}

Et ensuite, à Paris ?

\section{Jean-Pierre Vernant}

Et, au lycée à Paris, alors, où j'étais entre 1946 et 1948, j'avais un programme beaucoup plus strict qui était la préparation au baccalauréat : économie, morale et philosophie générale, mais pas d'histoire de la philosophie. 


\section{José Otávio Guimarães}

Enfin, quel est le bilan de votre activité comme professeur de philosophie dans ces années-là ?

\section{Jean-Pierre Vernant}

Je crois que je considérais que mon travail de professeur de philosophie était de déstabiliser; de faire comprendre aux gosses des choses..., comme la notion, par exemple, de perception. Je leur expliquais que ce qu'ils croyaient avoir sous le nez, la chair, les murs et tout ça, c'était des constructions complexes, des messages qui étaient envoyés, filtrés et que les choses n'étaient pas, que les animaux ne les voyaient pas de la même façon. Au début, pour qu'il y ait des fenêtres qui s'ouvrent dans l'espace qui était le leur, ils se posaient un tas de questions, car le monde n'était pas aussi simple qu'ils le croyaient. Par conséquent, ils en venaient à un moment donné à se poser la question (et je considérais que ce n'était pas mon boulot), à se dire : qu'est-ce que je suis ? Et qu'est-ce que c'est qu'exister? Qu'est-ce que c'est que vivre? C'est pour ça, je crois, qu'ils aimaient bien la classe. Ça les secouait et ça les concernait. En plus, il y avait l'idéologie vichyste - famille, travail, patrie -, et sans en avoir l'air, je la démolissais totalement. Pour ce qui est de l'idéologie nazie, sans le dire, je montrais que c'était une monstruosité. Ils comprenaient tout ça. Et pendant le temps où j'ai été professeur, certains étudiants ont compris non seulement ce que je pensais, mais pour certains aussi ce que je faisais. 